A. R. Heller ${ }^{1}$ R. J. Litz ${ }^{1}$ D. Wießner ${ }^{2}$. C. Dammann ${ }^{1} \cdot$ R. Weissgerber ${ }^{1}$

O. W. Hakenberg ${ }^{2}$. M. P. Wirth ${ }^{2}$.T. Koch ${ }^{1}$

${ }^{1}$ Klinik und Poliklinik für Anaesthesiologie und Intensivtherapie,

Universitätsklinikum Carl Gustav Carus, Technische Universität, Harvard Medical

International Associated Institution, Dresden . ${ }^{2}$ Klinik und Poliklinik für Urologie,

Universitätsklinikum Carl Gustav Carus, Technische Universität,

Harvard Medical International Associated Institution, Dresden

\title{
Betriebswirtschaftliche
}

\section{Auswirkungen der thorakalen Epiduralanästhesie bei urologischen Operationen}

\author{
Dargestellt am Beispiel der G-DRG M01B, OPS-301 5-604.0 \\ (radikale retropubische Prostatektomie)
}

D e Einführung des fallpauschalierten Entgeltsystems für den stationären Klinikbereich (G-DRG) eröffnet im deutschen Gesundheitssystem mit dem Ziel der Reduktion kostenintensiver Überkapazitäten einen vorher nie da gewesenen Verdrängungswettbewerb $[14,19,20]$. Entsprechend gewinnen ökonomische Aspekte bei den Leistungserbringern erheblich an Bedeutung [7, 9]. Dabei liegt Optimierungspotenzial nicht nur in den eigentlichen ärztlichen Teilprozessen, sondern v. a. auf der Ebene der Prozessorganisation und der nichtärztlichen „(Service-) Leistungen“ am Patienten [14, 19]. Folgerichtig integrieren nun auch Leistungserbringer zunehmend längst überfällige betriebswirtschaftliche Aspekte im Gesundheitssystem, wie Erlös- und Qualitätssicherung, Kostensenkung und Wettbewerb, in ihre Strategie zur Positionierung am „Markt“ $[6,7]$. Dies gilt für den Wettbewerb zwischen den Krankenhäusern ebenso wie für die Konkurrenz der Fachabteilungen innerhalb der Kliniken (und Klinikkonzerne) um Leistungszuweisungen $[14,19]$. Dabei kommen einer transparenten internen Leistungsverrechnung [23], der Etablierung einer effektiven interdisziplinären Kommunikationskultur [6] und einem strategischen Controlling [3, 7, 14], ggf. unter Einsatz automatisierter Prozesskontrollverfahren, Schlüsselstellungen $\mathrm{zu}[16,20]$.

Die Erlösseite kann in der gegenwärtigen Situation durch eine optimierte und patientenorientierte Prozess- und Arbeitsorganisation gestärkt werden $[4,14,16]$ und zu einer Steigerung des Patientendurchlaufs und damit der Fallzahlen führen. Eine Marktstrategie auf Basis der Steigerung der Fallgewichte dürfte hingegen, gerade im oberen Schweregradbereich, durch überproportionale Kosten limitiert sein. Insbesondere im Hinblick auf die lokale Marktpositionierung erscheinen Strategien mit dem Ziel der „Kundenorientierung " und der Steigerung der Patientenzufriedenheit langfristig Erfolg versprechen$\operatorname{der}[13,17]$.

Auf der Kostenseite müssen sowohl lohnende Einspar- $[8,30]$ als auch lohnende Investitionspotenziale $[3,9,15,25]$ identifiziert werden. Dazu gehört die Überprüfung gegenwärtiger Prozesse und Verfahren auf Kosteneffektivität, sowohl unter dem Sach- als auch unter dem Personalkostenaspekt [8]. Dabei kann eine sachmittelbezogen günstig erscheinende Prozedur aufgrund hierdurch vermehrt anfallender
Personalkosten eine negative Teilprozessbilanz verursachen [8]. Umgekehrt muss ein wertschöpfender kostenintensiver Teilprozess nicht zwangsläufig die Gesamtbilanz ungünstig beeinflussen [29]. Berücksichtigt man weiterhin, dass die Personalkosten in der Anästhesie mit 70\% den $\mathrm{Me}$ dikamenten- und Verbrauchsmitteln mit einem Anteil von nur 20\% im Budget gegenüberstehen $[1,26]$, wird klar, dass geringfügige Teilprozessoptimierungen mit Auswirkungen im Personalbereich größeren Einfluss auf das Betriebsergebnis haben als Sachmitteleinsparungen.

Im Rahmen moderner perioperativer Patientenpfade [4] besitzen die pathophysiologisch gut hinterlegten Effekte der thorakalen Epiduralanalgesie (TEA) [18] eine wesentliche Bedeutung für die perioperative Stresskontrolle mit der hieraus folgenden Reduktion von Mortalität und Morbidität $[5,12,25,27]$. Die günstigen Effekte neuer „Fast-track-Konzepte“ [27] können jedoch nicht allein der TEA zugeschrie-

Die Erhebung der Daten erfolgte als Teil der Promotionsarbeit von R. Weissgerber. Die Methodik der Isolierung verweildauerrelevanter Patientenfaktoren ist Teil der Promotionsarbeit von C. Dammann. 
ben werden, sondern sind nur im Konzert mit anderen Maßnahmen effektiv zu erzielen. Hierzu gehören neben festen Entlasskriterien und minimal-invasiven Techniken auch die frühe enterale Ernährung und die konsequente Mobilisierung. Welche Patientenfaktoren die Dauer des Krankenhausaufenthalts bestimmen, und inwieweit die präoperative Anlage eines thorakalen Epiduralkatheters und eine hiermit durchgeführte intra- und postoperative Schmerztherapie eine „lohnende Investition“ im Sinne des Gesamtprozesses sind und sich auf die Erlössituation auswirken, ist unklar [15].

Diese Arbeit identifiziert aufenthaltsund damit personal- sowie erlösrelevante Patientenmerkmale am Beispiel der OP-Prozedur 5-604.o (radikale retropubische Prostatektomie) und beschreibt den Prozessmehrwert durch den perioperativen Einsatz der TEA.

\section{Methoden}

Biometrische sowie anästhesie-, schmerztherapie- und abrechnungsrelevante Patientendaten [23] werden, ebenso wie perioperative Verlaufsdaten, in unserer Klinik mithilfe eines belegleserbasierten Erfassungssystems (ANDOK, DataPec, Pliezhausen) für alle Patienten prospektiv elektronisch dokumentiert. Alle Patienten, die sich im Zeitraum zwischen dem 01.01.2001 und dem 31.12.2002 einer Operation nach OPS-301 5-604.o (radikale retropubische Prostatektomie) unterziehen mussten, wurden in die dann retrospektive Datenbankanalyse einbezogen. Weitere postoperative Daten, die nicht durch die Anästhesiedatenbank abgebildet waren, wie z. B. postoperativer Transfusionsbedarf, Stuhlgang, Klinikverweildauer usw, wurden der Stationsdokumentation entnommen. Insgesamt wurden 57 Parameter bei 460 Patienten erhoben. Dabei ergaben sich 32 fehlende Werte (Vollständigkeit 99,88\%), für die „missing at random“ unterstellt wurde.

Zur Prüfung, welche unabhängigen $\mathrm{Pa}$ tientenfaktoren den Krankenhausaufenthalt im beobachteten Kollektiv mit welchem relativen Beitrag signifikant beeinflussen, wurden multifaktorielle Regressionsmodelle (- Tabelle 1) entwickelt. Die Vorbereitung der Daten erfolgte zunächst mit einer univariaten Analyse der statisti-

Tabelle 1

Perioperative Faktoren, die die Dauer des Krankenhausaufenthalts im Rahmen der OPS 5-604.0 modifizieren $(r=0,47)$

\begin{tabular}{|c|c|c|c|c|c|c|}
\hline \multirow{2}{*}{$\begin{array}{l}n=460 \\
\text { OPS 5-604.0 }\end{array}$} & \multicolumn{3}{|c|}{ Koeffizienten } & \multicolumn{3}{|l|}{ Statistik } \\
\hline & $\beta$ & Standard. $\beta$ & $p$ & Median & Quartile & $n(\%)$ \\
\hline (Modellkonstante) & 1,96 & - & - & - & - & - \\
\hline Reeingriff durchgeführt & 3,22 & 0,23 & 0,000 & 0 & $0-0$ & $14(3,0)$ \\
\hline $\begin{array}{l}\text { Tachykardie postoperativ } \\
\text { (ITS) 1-4 Episoden }\end{array}$ & 0,73 & 0,15 & 0,001 & 0 & $0-0$ & $102(22,2)$ \\
\hline Wundheilungsstörungen & 1,27 & 0,15 & 0,002 & 0 & $0-0$ & $42(9,1)$ \\
\hline Lebensalter & 0,06 & 0,13 & 0,004 & 65 & $61-68$ & - \\
\hline ASA-Status & 0,51 & 0,10 & 0,023 & 2 & $2-2$ & - \\
\hline Transfundierte EKs gesamt & 0,14 & 0,10 & 0,042 & 0 & $0-2$ & $186(40,4)$ \\
\hline Erster Stuhlgang [Tag] & 0,24 & 0,08 & 0,076 & 2 & $2-3$ & - \\
\hline $\begin{array}{l}\text { Postoperative pulmonale } \\
\text { Komplikationen }\end{array}$ & 0,79 & 0,07 & 0,099 & 0 & $0-0$ & $11(2,4)$ \\
\hline
\end{tabular}

Reihenfolge nach Modellgewicht (Betrag des standardisierten $\beta$-Koeffizienten). Koeffizienten multivariate Regressionsanalyse Median und Quartile. Reeingriff z. B. Neuanlage Blasenkatheter nach akzidentieller Entfernung ( $n=7)$, Lymphozelenrevision ( $n=3)$.

ITS Intensivtherapiestation; ASA American Society of Anesthesiologists; EK Erythrozytenkonzentrat.

Tabelle 2

Präoperative Faktoren, die die Dauer des Krankenhausaufenthalts im Rahmen der OPS 5-604.0 modifizieren $(r=0,26)$

\begin{tabular}{|lllllll}
\hline $\begin{array}{l}n=460 \\
\text { OPS 5-604.0 }\end{array}$ & \multicolumn{2}{l}{ Koeffizienten } & \multicolumn{3}{c}{ Statistik } \\
\hline (Modellkonstante) & $\boldsymbol{\beta}$ & Standard. $\boldsymbol{\beta}$ & $\boldsymbol{p}$ & Median & Quartile \\
\hline Lebensalter & 0,906 & - & - & - & - \\
\hline ASA-Status & 0,066 & 0,138 & 0,003 & 65 & $61-68$ \\
\hline Hb prä-OP [mmol/I] & $-0,290$ & $-0,093$ & 0,043 & 8,8 & $8,3-9,4$ \\
\hline NYHA-Status & 0,294 & 0,071 & 0,163 & 0 & $0-0$ \\
\hline
\end{tabular}

Reihenfolge nach Modellgewicht (Betrag des standardisierten $\beta$-Koeffizienten). Koeffizienten multivariate Regressionsanalyse, Median und Quartile.

ASA American Society of Anesthesiologists; $\mathrm{Hb}$ prä-OP präoperative Hämoglobinkonzentration; NYHA New York Heart Association.

schen Verteilungen der unabhängigen Variablen sowie der Zielparameter. Nominale unabhängige Variablen wurden in dichotome Variablen transformiert. Anschließend wurde eine bivariate Analyse der unabhängigen gegen die abhängigen Faktoren durchgeführt. Durch diese Maßnahmen wurden Linearität, Normalverteilung und Varianzgleichheit der Parameter gewährleistet. Multikolinearität wurde durch Entfernung jeweils einer von zwei Variablen aus dem Modell ausgeschlossen, die in einer Korrelationsmatrix aller unabhängigen Parameter eine Korrelation von mehr als 0,8 aufwiesen. Als
Kontrolle diente der Varianzinflationsfaktor (in allen Fällen $<1,7$ ). Fehlende Werte wurden nicht ersetzt. Anschließend erfolgte die rückwärts gerichtete multifaktorielle lineare Regressionsanalyse. Dabei wurden schrittweise immer diejenigen unabhängigen Faktoren aus dem Modell ausgeschlossen, deren Beitrag zum Gesamtmodell das Signifikanzniveau von o,1 überschritt. In die Analyse zu den perioperativen Einflussfaktoren auf den Gesamtaufenthalt wurden folgende Variablen einbezogen:

Präoperativ. Alter, Größe, Gewicht, Bodymass-Index, Blutdruck [systolisch, 


\section{Zusammenfassung}

Hintergrund. Ziele dieser Arbeit sind die Identifikation erlösrelevanter Patientenmerkmale am Beispiel der OP-Prozedur OPS 5-604.0 (radikale retropubische Prostatektomie) und die Beschreibung des Prozessmehrwerts durch den perioperativen Einsatz von thorakaler Epiduralanalgesie (TEA).

Methodik. Durch multifaktorielle Regressionsanalyse wurden Faktoren ermittelt, die die Krankenhausverweildauer bei $460 \mathrm{~Pa}$ tienten mit der Operation 5-604.0 in den Jahren 2001 und 2002 (prä-DRG) beeinflussten. Präoperativ vorliegende (Komorbiditäts-)Parameter dienten als Matchingfaktoren für eine "Matched-pair-Analyse" der TEA-Effekte.

Ergebnisse. Signifikant verweildauerrelevante Merkmale waren ASA-Status, Lebensalter, präoperative Hämoglobin-(Hb)
Konzentration, Auftreten postoperativer Tachykardien, Anzahl transfundierter Erythrozytenkonzentrate, Wundheilungsstörungen und Revisionseingriffe. Auf der Basis identischer Matchingkriterien konnten 27 Paare (mit/ohne TEA) gebildet werden. Während die TEA-Gruppe eine um $8 \pm 18$ min längere Anästhesieeinleitungszeit hatte, war die Narkoseausleitung um $3 \pm 9$ min verkürzt. Weder in der Anästhesiepräsenzzeit noch in den Anästhesie- oder Gesamtoperationskosten unterschieden sich die Paare signifikant, wohl aber in den Kosten für die postoperative Schmerztherapie. Hier war die TEA/patientenkontrollierte Epiduralanalgesie (PCEA) teurer. Demgegenüber war die postoperative Verweildauer unter TEA bei gleichzeitig verlängerter Schmerztherapiedauer verkürzt. Bei bestehender Operationsnachfrage und kon- sequenter Nutzung der TEA können 32 Prozeduren/Jahr zusätzlich durchgeführt werden. Die Umsatzrendite verbesserte sich durch die TEA um 2,7\%.

Schlussfolgerung. Kombinationsanästhesieverfahren sind auf den ersten Blick mit einem höheren Personal- und Sachaufwand verbunden. Bei genauer Analyse erweist sich diese Investition als lohnend, denn durch verkürzte Verweildauern und optimierte Schmerztherapie ergeben sich eine erhöhte „Kundenzufriedenheit" und ein erhebliches Mehrerlöspotenzial.

\section{Schlüsselwörter}

Krankenhausverweildauer .

Matched-pair-Analyse · Patientenpfad .

Prozessoptimierung .

Radikale Prostatektomie .

Thorakale Epiduralanalgesie

\section{Impact of thoracic epidural analgesia on revenue for G-DRG M01B, OPS-301 5-604.0 (radical retropubic prostatectomy)}

\begin{abstract}
Introduction. Using the surgical procedure OPS 5-604.0 (radical retropubic prostatectomy) as an example, our study identifies revenue-relevant patient characteristics and describes the impact of the perioperative application of thoracic epidural analgesia (TEA).

Methods. Factors affecting duration of stay were determined in 460 patients undergoing OPS 5-604.0 in the year 2001 and 2002 using multifactorial regression analysis. Preoperative parameters served as factors for matched-pair analysis of the effects of TEA. Results. Characteristics significantly affecting length of postoperative hospital stay were ASA status, age, preoperative
\end{abstract}

haemoglobin concentration, postoperative tachycardia, number of transfused packed red cells, wound infection and surgical revision. Based on identical matching criteria 27 pairs (with/without TEA) could be formed. While the induction time in the TEA group was $8 \pm 18 \mathrm{~min}$ longer $(p=0.04)$, emergence was briefer by $3 \pm 9 \min (p=0.045)$. Neither anaesthesia presence time nor anaesthesia costs or total costs of surgery differed significantly between the pairs. However, duration of epidural postoperative pain therapy was longer with TEA but in contrast, the postoperative length of hospital stay after TEA was reduced. Assuming a continuous demand for OPS 5-604.0 procedures, TEA enables 32 more procedures to be carried out per year with an increased yield on turnover of $2.7 \%$. Conclusion. At first sight combined anaesthesia procedures require more human resources and material, however, as a result of shortened hospital stay and optimized pain therapy patient satisfaction increases and a substantial potential for increased revenue is gained.

\section{Keywords}

Clinical pathway - Length of hospital stay .

Matched-pair analysis - Process adjustment .

Radical prostatectomy .

Thoracic epidural analgesia 
diastolisch, mittlerer arterieller Druck (MAP)], Hämoglobin- (Hb-)Konzentration, Gesundheitsstatus nach Klassifikation der American Society of Anesthesiologists (ASA) und Herzinsuffizienzschwere [NewYork-Heart-Association- (NYHA-)Status]. Anamnestisch erhoben: Diabetes mellitus, "chronic obstructive pulmonary disease“ (COPD), restriktive Ventilationsstörung, Hypertonie, Thromboembolien, Summe der Vorerkrankungen.

Intraoperativ. Operationsdauer, Tachy/Bradykardien, Hyper/-Hypotonien, Blutverlust, Transfusion von Erythrozytenkonzentraten.

Postoperativ. Hb-Konzentration, postoperative Transfusion von Erythrozytenkonzentraten, Tachy-/Bradykardien, Hyper/-Hypotonien, erster Stuhlgang, Ernährungsbeginn, Dauer der Schmerztherapie, Revisionsoperation, Wundheilungsstörungen, kardiale oder pulmonale Komplikationen, Thromboembolie.

Da ausschließlich die präoperativen Faktoren gegen die Effekte der Epiduralanästhesie auf postoperativer Ereignisse robust sind, erfolgte zur Festlegung der Matchingkriterien eine weitere Modellrechnung, in die lediglich die dargestellten präoperativen Variablen einbezogen wurden

(• Tabelle 2). Für den Vergleich der Patientenpfade mit und ohne TEA wurden jeweils Patientenpaare („matched pairs") gebildet, die in allen präoperativen signifikant verweildauerrelevanten Parametern (- Tabelle 2) identische Merkmalsausprägungen aufwiesen.

Zur Vermeidung eines Selektionsbias wurden von den 37 Patienten ohne TEA im Beobachtungszeitraum zunächst diejenigen 4 Patienten ausgeschlossen, bei denen absolute Kontraindikationen gegen eine TEA bestanden [10]. Sechs Patienten, davon einer ohne TEA, wurden am 14.08.2002 nach radikaler Prostatektomie im Rahmen der Klinikevakuierung aufgrund des Elbehochwassers 2002 ungeplant entlassen und aus der Analyse ausgeschlossen. Den verbleibenden Patienten wurde jeweils ein Partner mit TEA aus der Datenbank zugeteilt, der in allen statistisch signifikanten präoperativen Faktoren (• Tabelle 2) übereinstimmte. Für 5 Patienten fand sich im Beobachtungszeitraum kein Matchingpartner, entsprechend wurde kein Paar gebildet. In 2 Fäl-
Tabelle 3

Patientencharaktaristika [Grundgesamtheit $(n=460)$ und "matched pairs" $(n=2 \times 27)]$ bei OPS 5-604.0

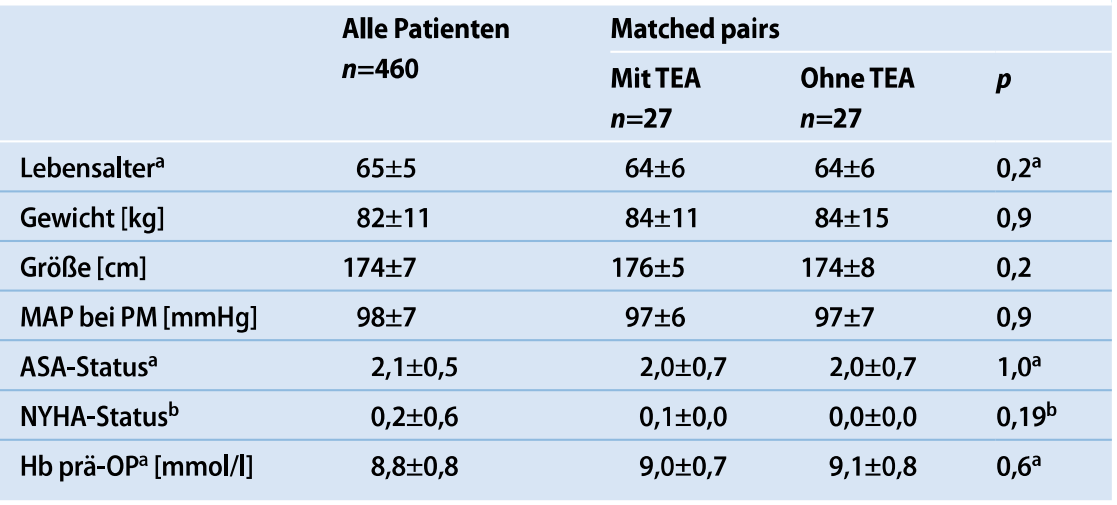

${ }^{a}$ Matchingkriterien It. - Tabelle 2. ${ }^{\text {b }}$ NHA-Status wurde nur dann zum Matching herangezogen, wenn mehr als ein Partner zur Auswahl stand.

MAP arterieller Mitteldruck, PM Prämedikation, ASA American Society of Anesthesiologists, NYHA New York Heart Association, Hb prä-OP präoperative Hämoglobinkonzentration, TEA thorakale Epiduralanalgesie.

\section{Tabelle 4}

Unterschiede zwischen Kombinationsanästhesie/Epiduralanalgesie (AA/TEA) und reiner Allgemeinanästhesie mit anschließender patientenkontrollierter intravenöser Opioidanalgesie (AA/PCIA); ,matched pairs" $(n=2 \times 27)$ bei OPS $5-604.0$

\begin{tabular}{|c|c|c|c|c|}
\hline & AA/TEA & AA/PCIA & $\begin{array}{l}\text { Gepaarte } \\
\text { Differen- } \\
\text { zen }^{a}\end{array}$ & $p$ \\
\hline Anästhesievorbereitungszeit [min] & $22 \pm 13$ & $19 \pm 12$ & $+3 \pm 19$ & 0,4 \\
\hline Anästhesieeinleitungszeit [min] & $38 \pm 12$ & $30 \pm 20$ & $+8 \pm 18$ & 0,038 \\
\hline Schnitt-Naht-Zeit [min] & $160 \pm 43$ & $161 \pm 46$ & $0 \pm 61$ & 1,0 \\
\hline Anästhesieausleitungszeit [min] & $5 \pm 4$ & $8 \pm 7$ & $-3 \pm 9$ & 0,045 \\
\hline Anästhesiezeit [min] & $244 \pm 48$ & $236 \pm 50$ & $+10 \pm 62$ & 0,4 \\
\hline Postanästhesiezeit ${ }^{\mathrm{b}}$ [min] & $17 \pm 10$ & $16 \pm 9$ & $+1 \pm 13$ & 0,7 \\
\hline Anästhesiepräsenzzeit [min] & $282 \pm 52$ & $271 \pm 50$ & $+15 \pm 68$ & 0,4 \\
\hline Anästhesiequalifikation des Mitarbeiters ${ }^{c}$ & $1,3 \pm 0,6$ & $1,4 \pm 0,7$ & $0 \pm 1$ & 0,6 \\
\hline Anästhesiekosten ${ }^{\mathrm{d}}[\mathrm{EUR}]$ & $489 \pm 87$ & $456 \pm 83$ & $+33 \pm 116$ & 0,15 \\
\hline OP-Kosten gesamt ${ }^{\mathrm{e}}$ [EUR] & $1.970 \pm 213$ & $1.914 \pm 202$ & $+55 \pm 274$ & 0,3 \\
\hline Postoperative Tachykardien [Episoden] & 1 & 2 & & 0,2 \\
\hline KH-Verweildauer postoperativ [Tag] & $6,6 \pm 1,4$ & $8,2 \pm 2,7$ & $-1,6 \pm 2,8$ & 0,007 \\
\hline KH-Verweildauer gesamt [Tag] & $9,1 \pm 1,7$ & $10,4 \pm 3,1$ & $-1,3 \pm 3,3$ & 0,04 \\
\hline $\begin{array}{l}\text { Kosten Normalstation inklusive Hotellerie } \\
\text { [EUR] }\end{array}$ & $1.702 \pm 303$ & $1.934 \pm 534$ & $-232 \pm 568$ & 0,04 \\
\hline $\begin{array}{l}\text { Kosten Schmerztherapie (Sachkosten) } \\
\text { [EUR] }\end{array}$ & $42 \pm 13$ & $12 \pm 4$ & $+30 \pm 13$ & $<0,001$ \\
\hline Kosten Gesamt [EUR] & $3.817 \pm 408$ & $4.005 \pm 537$ & $-188 \pm 676$ & 0,16 \\
\hline
\end{tabular}

${ }^{a}$ Matchingkriterien lt. - Tabelle 2, ${ }^{b}$ Post-Anästhesiezeit/Übergabe = Ende Anästhesie bis Ende Anästhesiepräsenz [23], ' Anästhesiequalifikation des Mitarbeiters: $0=$ AiP, $1=$ Assistenzarzt, $2=$ Facharzt $\mathrm{d}$ Innerbetriebliche Verrechnung aufPräsenzmin utenbasis (1,68 EUR/min; (1,21)) zuzüglich Sachkosten (EDA+Abwaschset). " Sach- und Personalkosten inklusive Anästhesie (Gleichzeitigkeitsschlüssel. s. Abschn. "Methoden"). 


\section{Originalien}
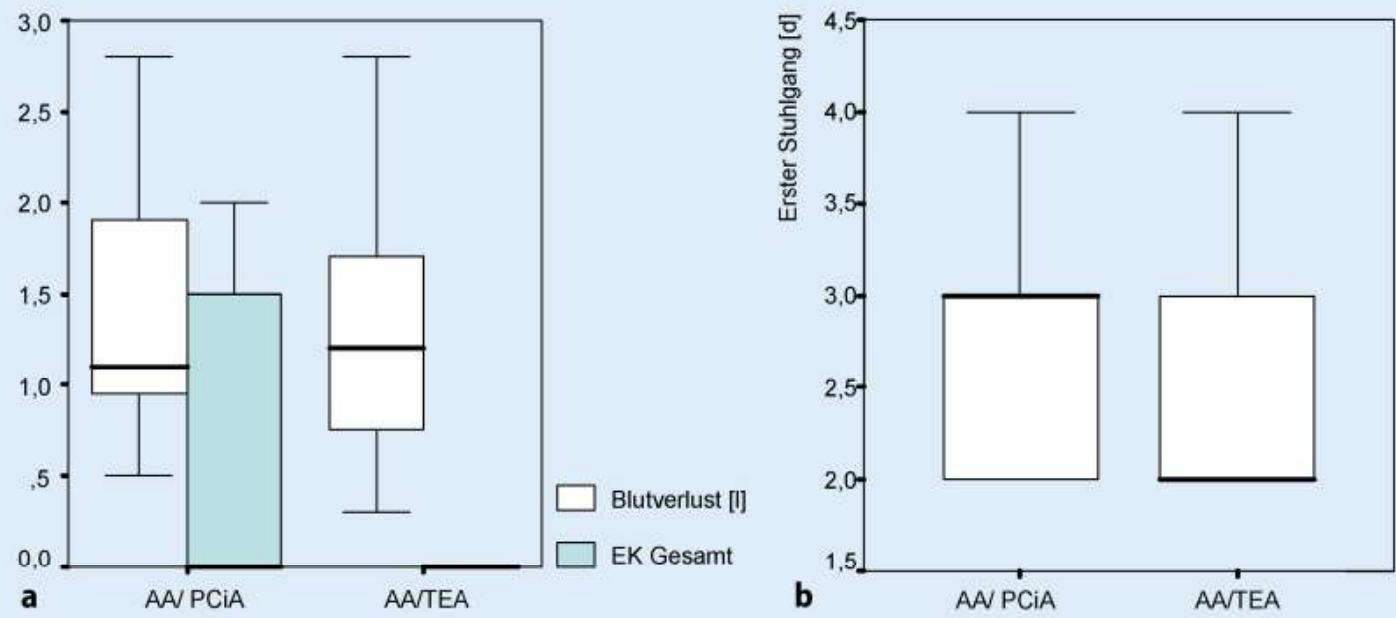

Abb. $1<$ Boxplot. a weiß Blutverlust [l], grau transfundierte Erythrozytenkonzentrate (Einheiten/ $300 \mathrm{ml}$ ). b Zeitpunkt des ersten Stuhlgangs unter Kombinationsanästhesie/Epiduralanalgesie (AA/TEA) oder mit reiner Allgemeinanästhesie und anschließender patientenkontrollierter intravenöser Opioidanalgesie $(A A / P C I A)$; , matched pairs" $(n=2 \times 27)$ bei OPS 5-604.0

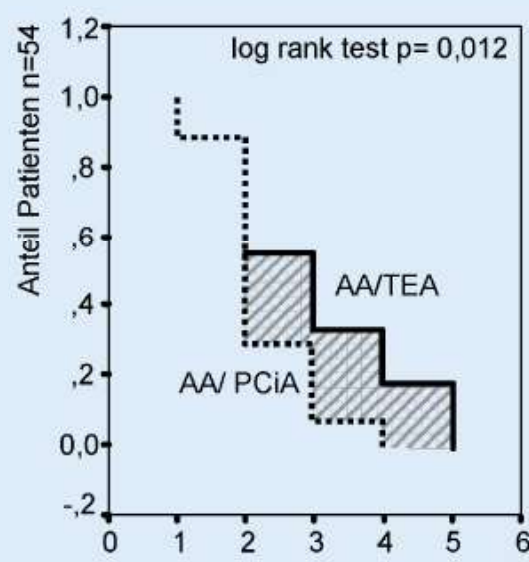

a Postoperative Schmerztherapie [d]

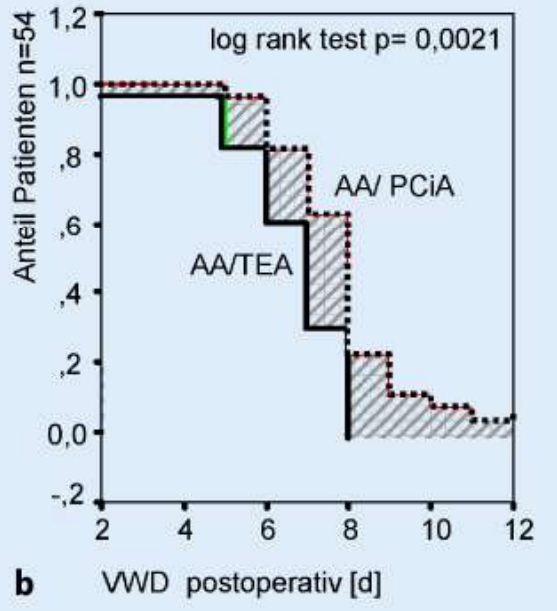

b WWD postoperativ [d]
Abb. $2 \varangle$ Mehrwert durch Epiduralanästhesie für Patient und Krankenhaus: Kaplan MeierAnalyse. a Dauer der Schmerztherapie schraffiert "Patienten-Komfortzone" und $b$ postoperative Krankenhausverweildauer (VWD), schraffiert ",Gewinnzone" mit Kombinationsanästhesie (AA/TEA) oder mit reiner Allgemeinanästhesie und anschließender patientenkontrollierter intravenöser Opioidanalgesie (AA/PCIA); "matched pairs" $(n=2 \times 27)$ bei OPS 5-604.0 len standen 2 Partner mit TEA zur Verfügung. Hier erfolgte die Zuordnung desjenigen Partners, der im regressionsanalytisch nächstrangigen (Betrag des standardisierten $\beta$-Koeffizienten) biometrischen Parameter am besten übereinstimmte. Auf diese Weise konnten 27 ,Zwillingspaare“ gebildet werden.

Grundannahmen für die Standard-Fallkosten-Analyse wurden aus einer hausinternen Wirtschaftlichkeitsanalyse für die Hüftgelenkendoprothetik übernommen [24]. Die Gesamtbasiskosten (Hotellerie, Technik, Energie usw.) lagen bei EUR 89,01/ Tag. Die Standardkosten für Sekundärdienstleistungen (EKG, Labor, Schreibdienst usw.) lagen bei EUR 62,50/Fall. Kosten für ärztliche und pflegerische Maßnahmen auf der Normalstation, einschließlich Anästhesie- und Schmerzvisiten, sowie zuordenbare und nichtzuordenbare Sachkosten wurden analog [24] berechnet: Kos ten $_{\text {NormStat }}=85,229^{\star}$ Tage $+114,57$ [EUR] $\left(r^{2}=0,9979\right)$. Dabei wurden interne Perso-
nal-Standard-Kosten [EUR/min] wie folgt zugrunde gelegt: Operateur 0,74; Anästhesist 0,64; Funktionsdienst im Operationsbereich o,41; Funktionsdienst der Anästhesie 0,43. Rüstzeiten Operationsfunktionsdienst Gleichzeitigkeitsfaktor 2,5: Beginn Anästhesie (BAN)-Beginn chirurgische Maßnahmen (BCM) + 15 min (abrüsten). Rüstzeit Operateure BAN-Schnitt einfach. Operative Wertschöpfungszeit (SchnittNaht-Zeit) Operateure 3 fach, Operationsfunktionsdienst 2 fach. Standardsachkosten-Operation (Abschreibung Investmittel, Instrumentarium, Verbrauchsmittel) wurden mit EUR 850/Fall erfasst. Die Verrechnung einer Anästhesieminute erfolgte, wie auch für die interne Leistungsverrechnung, auf Präsenzminutenbasis mit EUR 1,68/min [entspricht EUR 2,02/min reine Anästhesiezeit (RAnZ)]. Die Sachkosten für TEA-Set, einschließlich Abwaschset, betrugen EUR 13,12; Erythrozytenkonzentrat, einschließlich Kreuzprobe, EUR 79,00. Die Schmerztherapiesachkos- ten (einschließlich Geräteabschreibung) wurden für die patientenkontrollierte intravenöse Opioidanalgesie (PCIA; Piritramid) mit EUR 5,35/Tag und für die patientenkontrollierte Epiduralanalgesie (PCEA (Baxter i-pump); o,2\%iges Ropivacain+ $0,5 \mu \mathrm{g} / \mathrm{ml}$ Sufentanil ohne Basalrate; 5-mlBolus „lock out“ 20 min) mit EUR 13,00 + EUR 9,83/Tag kalkuliert [28]. Für die Umsatzerlösberechnungen wurde einheitlich der G-DRG-Fallpauschalenkatalog des Bezugsjahres 2004 herangezogen.

Die „Matched-pair-Analyse“ erfolgte bei intervall- oder ordinalskalierten Parametern mit dem zweiseitigen WilcoxonTest für verbundene Stichproben. Bei allen statistischen Vergleichen wurde die Nullhypothese $\mathrm{ab}$ einer Irrtumswahrscheinlichkeit von $p<0,05$ abgelehnt. Zur deskriptiven und explorativen Datenanalyse sowie für die Erstellung der Regressionsmodelle wurde das Programmpaket SPSS für Windows (Release 10.0.5, SPSS Inc., Chicago, IL, USA) verwendet. 


\section{Ergebnisse}

Im 2-jährigen Beobachtungszeitraum wurden an unserer Klinik 460 radikale retropubische Prostatektomien (OPS 5-604.0) durchgeführt. Biometrische Daten können der $\bullet$ Tabelle 3 entnommen werden. Allen Patienten wurde beim Prämedikationsgespräch eine Kombinationsanästhesie (TEA + Allgemeinanästhesie) für den Eingriff angeboten. Insgesamt erfolgten in den 2 Jahren des Beobachtungszeitraums 423 Eingriffe (OPS 5-604.0) in Kombinationsanästhesie und 37 in reiner Allgemeinanästhesie. In 25 Fällen wurde ein Epiduralkatheter abgelehnt, in 4 Fällen bestanden absolute Kontraindikationen [10] und in 8 Fällen wurde aufgrund ungünstiger anatomischer Verhältnisse an der Wirbelsäule entweder primär auf eine Punktion verzichtet oder diese im Verlauf abgebrochen. Alle Patienten mit frustranem TEA-Anlage-Versuch wurden aus der Analyse ausgeschlossen. Wurde keine TEA durchgeführt, erfolgte die postoperative Schmerztherapie in allen Fällen mit PCIA. Die TEA wurde immer während der Anästhesieeinleitungszeit angelegt [12, 23].

Aus den im Methodenteil definierten perioperativen Parametern wurden bei den 460 Patienten im Beobachtungszeitraum die Notwendigkeit eines Revisionseingriffs (mittlere Verlängerung der Verweildauer um 3,2 Tage/Reoperation), das Auftreten postoperativer Tachykardien (+o,7 Tage/tachykarde Episode), Wundheilungsstörungen $(+1,3$ Tage), das Lebensalter (+o,6 Tage/10 Jahre), ASA-Status (+o,5 Tage/ASA-Stufe) sowie die Anzahl im Gesamtverlauf transfundierter Erythrozytenkonzentrate [0,1 Tage/EK (300 ml)] als signifikant für die Dauer des Krankenhausaufenthalts identifiziert (• Tabelle 1). Bei insgesamt 460 Patienten waren in 14 Fällen Reeingriffe erforderlich (10 transurethrale Katheterneueinlagen nach spontaner Dislokation, 3 Punktionen pelviner Lymphozelen, 1 Hämatomrevision); kein Patient starb perioperativ.

Zur Bildung von Zwillingspaaren, deren Vergleich Rückschlüsse auf die Effekte einer TEA zulässt, wurde eine weitere Modellanalyse durchgeführt, die lediglich präoperative Faktoren einschloss. Hier durch wurde sichergestellt, dass Effekte der Epiduralanästhesie nicht das Mat-

Tabelle 5

Vergleichsbasis durchschnittliche Verweildauer in Deutschland nach G-DRG-Fallpauschalenkatalog 2004

\begin{tabular}{lllll} 
KH-Verweildauer It. Fallpauschalenkatalog & $\begin{array}{l}\text { Mittlere } \\
\text { Verweildauer }\end{array}$ & \multicolumn{2}{l}{ Grenzverweildauer } \\
& 18,1 & 5 & 31 \\
\hline M 01 A (mit äußerst schwerer Komorbidität) & Obere \\
\hline M 01 B (ohne äußerst schwere Komorbidität) & 14,6 & 4 & 25 \\
\hline
\end{tabular}

Tabelle 6

Fallbezogene Mehrkosten-Erlös-Rechnung für intraoperative

Kombinationsanästhesie/postoperative Epiduralanalgesie mit rein ärztlich

betriebener Schmerztherapie (ST Arzt) oder ärztlich supervidiertem Einsatz von patientenkontrollierter Epiduralanalgesie (PCEA)

\begin{tabular}{|lcllll} 
& \multicolumn{2}{c}{ Mehrkosten } & \multicolumn{2}{l}{ Erlöse } & \\
& ST-Arzt & ST-PCEA & M01B & Verweildauer \\
\hline Kombinationsanästhesie (Operation) & 32,67 & 32,67 & - & - \\
\hline Schmerztherapie $^{\text {a }}$ (postoperativ) & 297,53 & 46,08 & - & - \\
\hline Zwischensumme & 330,20 & 78,75 & $6.959,00$ & $-1,3$ Tage $(-14,3 \%)$ \\
\hline Gegenwert der VWD-Verkürz. & - & - & - & 994,14 \\
\hline Rest-Mehrwert TEA/Fall & 663,94 & 915,39 & - & - \\
\hline
\end{tabular}

a Schmerztherapiekosten (Sach- und Personalmittel) für $72 \mathrm{~h}$ berechnet auf Basis von Sebastian et al. [28]. ${ }^{\text {b }}$ Baxter i-pump mit Naropin-200 ml bag (AstraZeneca) +Sufentanil 0,5 $\mu \mathrm{g} / \mathrm{ml}$.

Tabelle 7

Gegenüberstellende Hochrechnung der möglichen Mehreingriffe/Jahr durch den Einsatz intraoperativer Kombinationsanästhesie und postoperativer Epiduralanalgesie je 230 DRGs M01B (Jahresmittel UKD 2001/02)

\begin{tabular}{|lcc|}
\hline $\begin{array}{l}\text { Prozeduren } \text { p.a } \\
\text { OPS 5-604.0 } \mathrm{n}=\mathbf{2 3 0} .\end{array}$ & $\begin{array}{c}\text { Verfahren } \\
\text { AA/TEA }\end{array}$ & AA/PCIA \\
\hline Krankenhausverweildauer [Tag] & 9,1 & 10,4 \\
\hline Pflegetage p.a. & 2.093 & 2.392 \\
\hline Differenz Patiententage p.a. [Tag] & 299 & - \\
\hline Mehr-Prozeduren mit AA/TEA & 32,8 & - \\
\hline
\end{tabular}

AA/TEA Kombinationsanästhesie/Epiduralanalgesie.

ching beeinflussen. Neben den bereits im Modell 1 ( $\bullet$ Tabelle 1) identifizierten Faktoren ASA-Status und Lebensalter, zeigte sich hier (• Tabelle 2) zusätzlich die präoperative Hb-Konzentration als signifikanter verweildauerrelevanter Einflussfaktor. Dabei führt eine um $1 \mathrm{mmol} / \mathrm{l}$ höhere $\mathrm{Hb}$ Konzentration zu einer Verkürzung des Aufenthalts um 0,3 Tage.

Im paarweisen Vergleich von Patienten mit und ohne TEA, die in signifikant verweildauerrelevanten präoperativen Faktoren identisch waren („matched pairs" nach - Tabelle 2) zeigten sich, bezogen auf die Anästhesiepräsenz (Beginn des Monito- rings im Einleitungsraum bis zur Übergabe auf der Wachstation), keine statistisch-signifikanten Unterschiede (• Tabelle 4). Teilprozesse, wie die Narkoseeinleitung (einschließlich zentraler Venenkatheter, invasive Blutdruckmessung) waren in der TEAGruppe um 8 min signifikant verlängert, während die Ausleitungszeit signifikant um 3 min verkürzt war. Präsenzminutenbasiert [23] und unter Berücksichtigung der zusätzlichen Sachkosten (Abwasch- und Nadel-Katheter-Set) konnte im Rahmen der TEA kein statistisch signifikanter Unterschied in den Anästhesiekosten nachgewiesen werden. Auch die Einbeziehung der Per- 
Tabelle 8

Hochrechnung DRG-bezogene Gewinnerzielung/Jahr durch den

konsequenten Einsatz intraoperativer Kombinationsanästhesie und

postoperativer Epiduralanalgesie unter Vernachlässigung von Budget-

begrenzungsmechanismen im stationären Krankenhausbereich

\begin{tabular}{|c|c|c|c|c|c|}
\hline \multirow{2}{*}{$\begin{array}{l}\text { Mögliche Prozeduren } \\
\text { OPS 5-604.0 innerhalb } \\
\text { eines Jahres }\end{array}$} & \multicolumn{2}{|c|}{ AA/TEA $(n=262)$} & \multicolumn{2}{|c|}{ AA/PCIA $(n=230)$} & \multirow[t]{2}{*}{ Differenz } \\
\hline & Kosten & Erlös & Kosten & Erlös & \\
\hline $\begin{array}{l}\text { Standardeinzelfall M01B } \\
\text { [EUR] }\end{array}$ & 3.817 & 6.959 & 4.005 & 6.959 & - \\
\hline Jahressumme [EUR] & 1.000 .172 & 1.823 .258 & 921.226 & 1.600 .570 & 222.688 \\
\hline Differenz (Gewinn) [EUR] & - & 823.086 & - & 679.344 & 143.742 \\
\hline Umsatzrendite [\%] & - & 45,1 & - & 42,4 & 2,7 \\
\hline
\end{tabular}

AA/TEA Kombinationsanästhesie/Epiduralanalgesie, AA/PCIA reine Allgemeinanästhesie mit anschließender patientenkontrollierter intravenöser Opioidanalgesie.

sonalkosten der Operateure und des Operationsfunktionsdienstes führte nicht $\mathrm{zu}$ einer signifikanten Kostensteigerung, die der TEA zugeordnet werden konnte.

Der in der TEA-Gruppe im Mittel um $192 \pm 1192 \mathrm{ml}$ reduzierte Blutverlust erforderte seltener die Transfusion von Erythrozytenkonzentraten (• Abb. 1a). Dieser Unterschied erreichte jedoch in der beobachteten Kohorte keine statisti sche Signifikanz. Ebenso war unter TEA früher eine Normalisierung des gastrointestinalen Transports zu verzeichnen (- Abb. 1b).

- Abbildung 2 zeigt die Effekte von TEA auf die Schmerztherapiedauer und den postoperativen Krankenhausaufenthalt. Bei gleichzeitiger Verlängerung der speziellen Schmerztherapie durch die TEA, die um EUR $30 \pm 13(p<0,001)$ teurer war als die PCIA, war die postoperative Entlassung der Patienten im Mittel um $1,6 \pm 2,8$ Tage signifikant früher möglich $(p=0,007)$. Aus der Verkürzung der Gesamtverweildauer um 1,3 $\pm 3,3$ Tage ergaben sich um EUR $232 \pm 568$ signifikant geringere Kosten für den Normalstationsaufenthalt $(p=0,04)$. Im Vergleich dazu weist der G-DRG-Browser des Instituts für das Entgeltsystem im Krankenhaus (InEK) für diesen Eingriff bundesweit Anästhesiekosten in Höhe von EUR 828 (12\% der Kosten), Operationsgesamtkosten, einschließ lich Anästhesie von EUR 2.867 (41\% der Kosten) und Normalstationskosten von EUR 2.560 (37\% der Kosten) aus. Die Ver weildauer nach G-DRG-Vergleichsbasis 2004 ist in $\bullet$ Tabelle 5 aufgeführt.
In Abhängigkeit davon, ob die postoperative Schmerztherapie mit einfachen kontinuierlich arbeitenden Spritzenpumpen, die eine bedarfsweise Bolusapplikation durch den Patienten nicht erlauben, und der ärztliche Schmerzdienst selbst Boli applizieren muss, oder ob andererseits - ärztlich supervidiert - eine PCEA zum Einsatz kommt, ergeben sich deutliche Kostenunterschiede zugunsten der PCEA, die in $\bullet$ Tabelle $\boldsymbol{6}$ in einer Modellrechnung über $72 \mathrm{~h}$ zusammengestellt sind. Bringt man die intraoperativen (statistisch nicht signifikanten) Mehrkosten in der TEA-Gruppe und entsprechende Schmerztherapiemehrkosten verfahrensspezifisch [28] in Ansatz, so fallen die in - Tabelle 6 links ausgewiesenen Mindererlöse an. Diesen steht aber eine Effizienzsteigerung des Gesamtprozesses OPS 5-604.o um 14,3\% (-1,3 Tage) gegenüber, dem bezogen auf die DRG Mo1B rechnerisch ein Gegenwert von EUR 994,14/Fall entspricht.

Die operative Jahresleistung von 230 Prozeduren der OPS 5-604.0/DRG Mo1B (• Tabelle 7) wird bei einer Verweildauer von 10,4 Tage (AA/PCIA) in 2.392 Pflegetagen erbracht. Wenn die Effizienzsteigerung des Prozesses durch die TEA (299 Pflegetage weniger) in $32 \mathrm{Mehr}$ prozeduren umgesetzt werden kann, so ist mit Umsatzmehrerlösen in Höhe von EUR 222.688,00 zu rechnen. Diese Umsatzsteigerung ( Tabelle 8) ist mit Mehrkosten von EUR 78.946 verbunden, sodass die Anwendung der TEA unter den gegebenen Bedingungen zu einer Gewinnsteigerung von EUR 143.742 führen kann. Hieraus ergibt sich eine hypothetische Umsatzrendite (Gewinn/Umsatzerlöse $\times 100$ [\%]) mit TEA von $45,1 \%$ und ohne TEA von $42,4 \%$.

\section{Diskussion}

Die patientenorientierte Optimierung von Arbeitsprozessen bei gleichzeitiger Steigerung von Qualität und Patientenzufriedenheit gewinnt spätestens seit Einführung des fallpauschalierten Entgeltsystems DRG vitales Interesse für die Krankenhausbetreiber [14, 19]. Untersuchungen der letzen Jahre zur multimodalen Optimierung operativer Behandlungspfade wurden überwiegend mit dem Ansatz betrieben, die medizinische Therapie $\mathrm{zu}$ verbessern $[5,12,27]$ oder Komplikationen zu vermeiden [25], nicht jedoch mit primär betriebswirtschaftlicher Intention [29]. Während sich „harte“ Qualitätsindikatoren, wie Krankenhausmortalität, im Rahmen von elektiven Routineeingriffen mit extrem niedriger Mortalität nicht als Messinstrumente eignen, müssen entsprechende „weiche“ Surrogatparameter, wie die Krankenhausverweildauer oder die Notwendigkeit von Revisionsoperationen, Patientenzufriedenheit usw., für die Beurteilung der Behandlungsqualität herangezogen werden [14]. Dabei muss aus betriebswirtschaftlicher Sicht nicht nur die Prozessqualität optimiert werden (Therapieverbesserung), sondern insbesondere die Ergebnisqualität mit Augenmerk auf die Kosten-Nutzen-Relation. Hierbei kann die Verweildauer ein valider Surrogatparameter für Prozesseffektivität und für den Organisationsgrad der Teilprozesse sein [20]. Somit ist die Verkürzung der Krankenhausverweildauer nur ein mittelbares (aber messbares) Ziel auf dem Weg der Prozessoptimierung.

Ziel dieser Untersuchung war es zu überprüfen, welche Faktoren ursächlich für eine Verlängerung der Krankenhausverweildauer im Rahmen eines definierten Prozesses (OPS 5-604.0, Mo1B) sind, und inwieweit sich die medizinisch mittlerweile als evident zu betrachtende Verbesserung des perioperativen Managements durch TEA $[5,12,13,25,27]$ betriebswirtschaftlich abbilden lässt.

Um die Daten praxisrelevant $\mathrm{zu}$ betrachten und untersucherbedingte Verzer- 
rungen zu vermeiden, die generell einen Studienbias zugunsten konventioneller Verfahren erzeugen [22], wurden routinemäßig erfasste Patientendaten als Grundlage für die vorliegende Analyse verwendet, weil nur diese gegen gezielte oder ungezielte Einflussnahmen (Studienbias) robust sind $[14,20]$. Darüber hinaus wurde ein Beobachtungszeitraum gewählt, in dem keine relevanten Verfahrens- oder Personalveränderungen stattfanden. Aus dem Datenpool erfolgte die Bestimmung interventionsunabhängiger verweildauerrelevanter Faktoren (- Tabelle 2), die als Kriterien für eine Matched-pair-Analyse herangezogen wurden.

Eine Matched-pair-Analyse erschien v. a. aus dem Grund die valideste Auswertungsstrategie für die vorhandene Datenbasis zu sein, weil die intra- und postoperative TEA in unserer Institution ein integrativer Bestandteil des interdisziplinären Patienten-Behandlungs-Pfades im Rahmen der OPS 5-604.0 ist. Entsprechend fordern mittlerweile auch die Operateure die TEA von uns ein $[32,33]$. Folglich wurde dieses Verfahren allen Patienten ohne Kontraindikationen angeboten, und die überwiegende Mehrzahl (91,3\%) hat davon Gebrauch gemacht. Im Lichte unserer eigenen Erfahrungen [12] hielten wir eine Randomisierung TEA vs. AA nicht für vertretbar. Dieser hohe Anteil an $\mathrm{Pa}$ tienten mit TEA erklärt schließlich auch, warum das Vorhandensein einer TEA in der multivariaten Analyse (• Tabelle 1) nicht als unabhängiger verweildauerrelevanter Faktor in Erscheinung tritt. Zur Vermeidung eines Selektionsbias zugunsten der TEA wurden Patienten, die aufgrund einer Kontraindikation keine TEA erhalten hatten, nicht in die Paarbildung einbezogen. Zur Analyse kamen schließlich nur solche Patientenpaare, die in hohem Maße miteinander vergleichbar waren; eine Situation, die in prospektiv randomisierten Untersuchungen üblicherweise so nicht hergestellt wird.

Aufgrund der antizipierbaren Schwere dieses elektiven Eingriffs und des protrahierten natürlichen Verlaufes der Erkrankung Prostatakarzinom kommen Patienten mit einem ASA-Status $\geq_{3}$ und einer erwarteten Restlebenserwartung von $<10$ Jahren für den Eingriff in der Regel nicht in Betracht. Entsprechend ist das Patienten- gut, das der untersuchten operativen Prozedur unterzogen wird, als solches vorselektiert und weist eine niedrigere Komorbidität als die alters- und geschlechtsgematchte Bevölkerungsgrundgesamtheit auf.

Kombinationsanästhesieverfahren sind auf den ersten Blick mit einem höheren Personal- und Sachaufwand verbunden, der sich jedoch bei strukturierter Betrachtung als statistisch nicht signifikant herausstellt und in Relation zu den Gesamtfallkosten eine untergeordnete Bedeutung besitzt (• Tabelle 4). Der Zeitmehraufwand für die Anlage einer TEA in der Narkoseeinleitung fällt zudem je nach Operationssaalbelegung und durchschnittlicher Schnitt-Naht-Zeit unter Umständen gar nicht ins Gewicht, da bei Operationsdauern über 120 min ohnehin pro Arbeitstag lediglich 3 Operationen durchgeführt werden können, unabhängig davon, ob die Wechselzeit 20 oder 40 min in Anspruch nimmt [3]. Gleichzeitig wird der zeitliche Mehraufwand der TEA in der Narkoseeinleitung durch verkürzte Aufwachzeiten [12] ( $\bullet$ Tabelle 4) und eine schmerzund tachykardiefreie (und damit „kundenfreundlichere") Postanästhesie- und Übergabezeit (Wachstation) kompensiert.

Zudem ergibt sich, abhängig vom Anästhesieverfahren, ein erhebliches Umsatz-Mehrerlös-Potenzial durch Reduktion von operativen Komplikationsraten und der Krankenhausverweildauer (• Tabelle 6,7 ). In diesem Zusammenhang spielt die Vermeidung postoperativer Darmatonien und paralytischem Ileus eine wesentliche Rolle, da durch diese Komplikationen durchschnittliche Mindererlöse in Höhe von US\$ 6.709/Patient entstehen [31]. Bei Patienten nach radikaler retropubischer Prostatektomie [12] und noch deutlicher nach Zystektomie mit Neoblase konnte die Inzidenz des postoperativen Ileus durch TEA um den Faktor 4 gesenkt werden, sodass die Normalisierung der Darmtätigkeit insgesamt 2 Tage früher zu verzeichnen war [33]. Ebenso war die Inzidenz verweildauerrelevanter Revisionseingriffe auf die Hälfte reduziert. Dementsprechend konnte unter TEA ein früherer enteraler Nahrungsaufbau erfolgen, der für die zeitnahe Wiederherstellung der Homöostase elementar ist. Reproduzierbar günstige Effekte der TEA ergeben sich hier v. a. im Konzert mit anderen Re- 
habilitationsmaßnahmen, wie konsequenter Mobilisierung, früher enteraler Ernährung und der Definition fester Entlasskriterien $[5,27]$.

Hinzu kommt ein weiterer Punkt: In der postoperativen Schmerztherapie gilt, nach wie vor, die PCIA als ,golden standard“. Gerade im Zusammenhang mit Eingriffen am Intestinum beeinträchtigt aber eine protrahierte Opiattherapie die Darmfunktion zusätzlich. Darüber hinaus konnte gezeigt werden, dass die TEA der PCIA bei vergleichbarer Analgesiequalität im Hinblick auf ihr Nebenwirkungsspektrum (Kopfschmerz, schmerzbedingte Atmungseinschränkung, inaktivierende Vigilanzminderung) signifikant überlegen ist und entsprechend eine frühzeitigere Mobilisierung erlaubt [32]. Gerade dieses Nebenwirkungsspektrum der PCIA könnte in der vorliegenden Untersuchung auch der signifikant verkürzten Schmerztherapiedauer (• Abb. 2a) in dieser Gruppe zugrunde gelegen und umgekehrt den Patientenkomfort in der TEA-Gruppe erhöht haben.

In der Zusammenschau beeinflusst die TEA im perioperativen Patientenmanagement eine Reihe von Faktoren im Sinne des Gesamtprozesses günstig, die gleichzeitig als verweildauerverlängernd und damit erlösmindernd identifiziert wurden (• Tabelle 1). Darüber hinaus steigern insbesondere diejenigen Eigenschaften der TEA, die die Dauer des postoperativen Dyskomforts und die Verweildauer verkürzen, die Akzeptanz des Verfahrens bei Patienten und Operateuren und erhöhen somit die Kundenzufriedenheit. Damit entsteht für alle Beteiligten (Patient/ Anästhesist und Operateur/Klinikträger) eine „win/win/win-Situation“.

Diese günstige Situation kann durch die Anwendung von PCEA betriebswirtschaftlich noch weiter optimiert werden (• Tabelle 6). Die initialen Investitionskosten solcher Systeme können sich durch Reduktion der Lokalanästhetikumbasalrate und kompensatorischer PCEA-Bolus-Applikation bezogen auf die Medikamentenkosten bereits nach 41 Patienten (mit 72-h-Schmerztherapie) bei gleichbleibender Analgesiequalität amortisieren. Gegenüber einer rein arztbasierten Bolusapplikation können PCEAPumpen unter Berücksichtigung der Personalkosten, je nach Dosierungsregime, sogar bereits nach 8-9 Patienten (mit 72-h-
Schmerztherapie) in die "Gewinnzone“ fahren [28] bzw. zur fallbezogenen Erlössicherung beitragen ( $\bullet$ Tabelle 6$)$.

Den medizinischen und betriebswirtschaftlichen Vorteilen der TEA steht das Risiko epiduraler Hämatome mit der Folge eines operativen Interventionsbedarfes und einer Querschnittslähmung als „worst case scenario“ gegenüber. Ältere Untersuchungen schätzten das diesbezügliche Risiko erheblich zu niedrig ein. Neuere Studien aus Frankreich [2] oder Schweden [21] berichten, abhängig vom operierenden Fachgebiet, von Inzidenzen zwischen 1:25.000 (Geburtshilfe) und 1:3.600 (Orthopädie). In unserem urologischen $\mathrm{Pa}$ tientengut wurde bei bislang 10.817 Epiduralkathetern kein epidurales Hämatom beobachtet [11]. Diese Beobachtung deckt sich mit der Daten von Moen et al. $(n=1.710 .000)$, der urologische Patienten im Gegensatz zu orthopädischen nach neuraxialen Blockaden eher in die Niedrigrisikogruppe für epidurale Hämatome einordnet [21].

Zieht man die Krankenhausverweildauer als ,weichen“"Qualitätsindikator [14, 20] für den Prozess OPS 5-604.0/ Mo1 B heran, so liegt die von uns beobachtete Krankenhausverweildauer (• Tabelle 4) deutlich unter der nationalen Benchmark (• Tabelle 5). Vergleicht man den TEAEinfluss auf die Gesamtverweildauer und die postoperative Verweildauer $(\bullet$ Tabelle 4) zeigt sich, dass die Effektgröße der ausschließlich intra- und postoperativ wirksamen TEA groß genug ist, die Gesamtverweildauer ebenfalls signifikant $\mathrm{zu}$ verkürzen. Damit ist dieses Verfahren in der Lage, zeitliche Auswirkungen noch optimierungsfähiger präoperativer Teilprozesse zu kompensieren. Insbesondere in der präoperativen Phase können prozedurenspezifische Patientenpfade [4] Arbeitsabläufe standardisieren und zur weiteren Prozessoptimierung beitragen.

\section{Fazit für die Praxis}

Kombinationsanästhesieverfahren sind auf den ersten Blick mit einem höheren Personal- und Sachaufwand verbunden, der jedoch in Relation zu den Gesamtfallkosten lediglich eine untergeordnete Bedeutung besitzt. Im Gegenteil ergibt die betriebswirtschaftliche Analyse ein er- hebliches Mehrerlöspotenzial durch die TEA aufgrund der Reduktion operativer Komplikationsraten sowie der Krankenhausverweildauer und damit eine Effizienzsteigerung des Gesamtprozesses. Bezogen auf die DRG M01B erlaubt die Anwendung der TEA bei gegebener Infrastruktur und Vernachlässigung von Budgetbegrenzungsmechanismen $14,3 \%$ mehr Prozeduren/Jahr, denen eine Steigerung des erzielbaren Gewinns von EUR 143.742 gegenüberstehen kann.

\section{Korrespondierender Autor \\ PD Dr. A. R. Heller DEAA}

Klinik und Poliklinik für Anaesthesiologie und Intensivtherapie,

Universitätsklinikum Carl Gustav Carus, Dresden E-Mail: axel.heller@mailbox.tu-dresden.de

\section{Danksagung}

Die Autoren danken Herrn Dipl. Ing. Günther Böhme, Klink für Anästhesiologie UKD, für die Pflege der ANDOK-Datenbank sowie für die Vorverarbeitung und Plausibilitätskontrolle der Daten, sowie Frau Simone Weigel, Bereichscontrolling KAI/UKD, für die Unterstützung bei der Kosten-Erlös-Kalkulation.

Interessenkonflikt: Der korrespondierende Autor versichert, dass keine Verbindungen mit einer Firma, deren Produkt in dem Artikel genannt ist, oder einer Firma, die ein Konkurrenzprodukt vertreibt, bestehen.

\section{Literatur}

1. Albrecht DM, Winzer WEB (Hrsg) (2003) Qualität und Strukturen - Jahresbericht 2003 des Universitätsklinikums Dresden, Dresden, Eigenverlag

2. Auroy Y, Narchi P, Messiah A, Litt L, Rouvier B, Samii $K$ (1997) Serious complications related to regional anesthesia: results of a prospective survey in France. Anesthesiology 87:479-486

3. Bender HJ, Waschke K, Schleppers A (2004) Tischlein wechsle dich: Sind Wechselzeiten ein Maß für ein effektives OP-Management? Anaesthesiol Intensivmed 9:529-535

4. Braun J, Walter M, Kuhly R et al. (2003) Clinical Pathways und Diagnosis-Related Groups: Die Anästhesiologie als Schnittstellenfach. Anaesthesiol Intensivmed 9:637-646

5. Brodner G, Aken H van, Hertle L et al. (2001) Multimodal perioperative management - combining thoracic epidural analgesia, forced mobilization, and oral nutrition - reduces hormonal and metabolic stress and improves convalescence after major urologic surgery. Anesth Analg 92:1594-1600

6. EiffW von (2000) Personalmanagement in deutschen Krankenhäusern. Führungsdefizite behindern Organisation und Mitarbeiterengagement. HNO 48:773-766 
7. Eiff W von (2001) Der Medizin-Manager - Die neue Rolle des Arztes. HNO 49:479-481

8. EiffW von, Ziegenbein R von (2000) Laparoskopische Einweg-Instrumente: Kostspieliges Outsourcing von Produktqualität? Zentralbl Gynakol 122:439-444

9. Geldner G, Eberhart LH, Trunk S, Dahmen KG, Reiss mann T, Weiler T, Bach A (2002) Effizientes OP-Management. Vorschläge zur Optimierung von Prozessabläufen als Grundlage für die Erstellung eines OP-Statuts. Anaesthesist 51:760-767

10. Gogarten W, Aken H van, Büttner J, Riess H, Buerkle H (2003) Rückenmarksnahe Regionalanästhesien und Thromboembolieprophylaxe/antithrombotische Medikation. Überarbeitete Leitlinien de Deutschen Gesellschaft für Anästhesiologie und Intensivmedizin. Anaesthesiol Intensivmed 3:218 230

11. Heller AR, Litz RJ (2005) Why do orthopedic patients have a higher incidence of serious complications after central neuraxial blockade? Anesthesiology 102:1286

12. Heller AR, Litz RJ, Djonlagic I, Manseck A, Koch T, Wirth MP, Albrecht DM (2000) Kombinierte Anästhesie mit Epiduralkatheter. Eine retrospektive Analyse des perioperativen Verlaufs bei Patienten nach radikaler Prostatektomie. Anaesthesist 49:949-959

13. Heller AR, Horter M, Horter S, Litz RJ, Koch T (2003) Quality of anaesthesia - A patient's view. Eur J Anaesthesiol 20 [Suppl 30]:4

14. Heller G, Swart E, Mansky T (2003) QualitätsanaIysen mit Routinedaten - Ansatz und erste Analysen aus dem Gemeinschaftsprojekt "Qualitätssicherung mit Routinedaten" (QSR). In: Klauber J, Robra BP, Schellschmidt H (Hrsg) Krankenhausreport 2003. Schattauer, Stuttgart, S 271-288

15. Koch T, Heller AR (2000) Pathophysiologische und ökonomische Aspekte bei Regionalanästhesieverfahren. Anaesthesiol Intensivmed 41:138-146

16. Konrad C, Schmeck J, Schüpfer G (2001) Statistische Prozesskontrolle zum Qualitätsmanagement in der Anästhesie. Anaesthesiol Intensivmed 42:946-950

17. Krieter H, Russ N, Denz C, Ackern K van (2003) Erwartungen und Wünsche anästhesiologischer $\mathrm{Pa}$ tienten: Welche Faktoren tragen zur Zufriedenheit bei? Anaesthesiol Intensivmed 1:43-49

18. Litz RJ, Bleyl JU, Frank M, Albrecht DM (1999) Kombinierte Anaesthesieverfahren. Anaesthesist 48:359-372

19. Mansky T (2000) Grundlagen der Fallorientierten Leistungsbewertung im Krankenhausvergleich und im Entgeltsystem: Bewertungsmodule des DRG-Systems am Beispiel der Medicare-Versicherung. In: Sieben G, Litsch M (Hrsg) Der Krankenhausbetriebsvergleich. Ein Instrument auf dem Weg zu leistungsorientierten Preisen im Krankenhausmarkt. Springer, Berlin Heidelberg New York, S 149-191

20. Mansky T (2004) Helios-Analyse: Die Verweildauer sinkt, der Wettbewerb wird härter. Führen Wirtsch Krankenh 21:212-219

21. Moen V, Dahlgren N, Irestedt L (2004) Severe neurological complications after central neuraxial blockades in Sweden 1990-1999. Anesthesiology 101:950-959

22. Müller M (2000) Fortschritte bei der Konzeption klinischer Arzneimittelprüfungen. Onkologie 23:487491

23. Raetzell M, Reissmann H, Steinfath $M$, Schuster $M$, Schmidt C, Scholz J, Bauer M (2004) Implementierung einer internen Leistungsverrechnung über Anästhesieminuten Anaesthesist 53:1219-1230
24. Reinicke K (2003) Von der Fallkostenkalkulation nach dem DRG-Kalkulationshandbuch zur Lenkungskonzeption für das Krankenhausmanagement anhand der primären Endoprothetik am Hüftgelenk in der Klinik und Poliklinik für Orthopädie des Universitätsklinikums Dresden. Akademie für Controlling, Management und Qualitätssicherung des Deutschen Vereins für Krankenhaus-Controlling, Berlin, S 1-37

25. Rodgers A, Walker N, Schug S et al. (2000) Reduction of postoperative mortality and morbidity with epidural or spinal anaesthesia: results from overview of randomised trials. BMJ 321:1493

26. Schleppers A, Sturm J, Bender HJ (2003) Implementierung einer Geschäftsordnung für ein zentrales OP-Management - Öl oder Sand im Getriebe des OP-Ablaufs? Anaesthesiol Intensivmed 4:295-304

27. Schwenk W, Raue W, Haase O, Junghans T, Muller JM (2004) „Fast-track-Kolonchirurgie“. Erste Erfahrungen mit einem "clinical pathway" zur Beschleunigung der postoperativen Rekonvaleszenz. Chirurg 75:508-514

28. Sebastian H, Heller AR, Wiessner D, Hupfer A, Koch T, Litz R (2004) Vergleich von Effizienz und Ökonomie dreier Techniken zur patienten-kontrollierten Epiduralanalgesie bei Patienten mit radikaler Prostatektomie. Abstractband Deutscher Anästhesiekongress, Diomed, Ebelsbach, S 98

29. Senkal M, Mumme A, Eickhoff U et al. (1997) Early postoperative enteral immunonutrition: clinical outcome and cost-comparison analysis in surgical patients. Crit Care Med 25:1489-1496

30. Snyder-Ramos SA, Bauer M, Martin E, Motsch J Böttiger BW (2003) Preislisten am anästhesiologischen Arbeitsplatz - Stärkung des Kostenbewusstseins durch Einbeziehung in Prozess- und Kostenoptimierungsmaßnahmen. Anaesthesist 52:154161

31. Swenson BR, Hollenbeak CS, Koltun WA (2002) Hos pital costs and risk factors associated with complications of the ileal pouch anal anastomosis. Surgery 132:767-773

32. Wiessner D, Litz RJ, Heller A, Wirth MP (2003) Beurteilung der Qualität der perioperativen Schmerztherapie bei urologischen Operationen anhand der Daten von 803 Patienten. Urolog Nachr 9 [Suppl 2]:10

33. Wiessner D, Litz RJ, Heller AR, Georgieff M, Leike $S$, Koch T, Wirth MP (2004) Continuous thoracic epidural anaesthesia reduces incidence and duration of postoperative ileus in patients undergoing radical cystectomy. Eur Urol Suppl 3:592 